\title{
Transatlantica
}

Revue d'études américaines. American Studies Journal

\section{Lee Kovacs, The Haunted Screen : Ghosts in Literature} and Film.

Jefferson and London : McFarland Publishers, 1999.

\section{Anne Luyat}

\section{(2) OpenEdition}

\section{Journals}

Édition électronique

URL : http://journals.openedition.org/transatlantica/341

DOI : 10.4000/transatlantica.341

ISSN : 1765-2766

Éditeur

AFEA

Référence électronique

Anne Luyat, "Lee Kovacs, The Haunted Screen: Ghosts in Literature and Film. », Transatlantica [En ligne], 1 | 2001, mis en ligne le 23 mars 2006, consulté le 29 avril 2021. URL : http://journals.openedition.org/ transatlantica/341; DOI : https://doi.org/10.4000/transatlantica.341

Ce document a été généré automatiquement le 29 avril 2021.

\section{c) (†) $९$}

Transatlantica - Revue d'études américaines est mis à disposition selon les termes de la licence Creative Commons Attribution - Pas d'Utilisation Commerciale - Pas de Modification 4.0 International. 


\title{
Lee Kovacs, The Haunted Screen : Ghosts in Literature and Film.
}

\author{
Jefferson and London : McFarland Publishers, 1999.
}

Anne Luyat

1 Entre le fantôme littéraire et sa représentation cinématographique, au cœur des espaces très différents hantés par l'un et par l'autre, Lee Kovacs nous donne rendez-vous. Une analyse minutieuse des transformations qui ont lieu dès que le fantôme quitte les pages du livre pour passer à l'écran est au cœur de l'essai, qui révèle la faiblesse des spectres contemporains par rapport à ceux d'antan. Au départ, le lecteur a le plaisir de retrouver une vieille connaissance, le lémure de Cathy qui hante les visions de Heathcliff dans Wuthering Heights (Emily Brontë et William Wyler) avec une puissance restée intacte depuis un siècle et demi. Un saut dans le temps fait apparaitre quatre revenants romantiques, encore vaillants quoique dépourvus d'une partie du pouvoir surnaturel des visions gothiques : le capitaine Gregg dans The Ghost and Mrs Muir (R.A. Dick et Joseph Mankiewicz), Jennie dans Portrait of Jennie (Robert Nathan et William Dieterle), la femme inconnue dans Letter from an Unknown Woman (Stephan Zweig et Max Ophuls) ainsi que Carmel dans The Uninvited (Dorothy Macardle et Lewis Allen). Si le terme de romantique n'est pas toujours bien défini-l'écart se creuse entre le livre et l'adaptation cinématographique - ces esprits ont un pouvoir maléfique, parfois charmant, qui surprend. Ensuite apparaissent les fantômes du théâtre porté à l'écran : Liliom dans Liliom (Ferenc Molnar et Fritz Lang), Emily Webb et les occupants du cimetière dans Our Town (Thornton Wilder et Sam Wood). Très différents de leurs homologues livresques, capables de gestes émouvants en dépit du temps relativement court où ils sont visibles, ils ne semblent pas gênés par le passage de la scène à l'écran. En revanche, les fantômes créés pour le cinéma, et privés ainsi de tout passé littéraire, ne sont que des ombres de leurs prédécesseurs gothiques et romantiques. Sans pouvoir surnaturel, sans domicile attitré, affaiblis au point de devoir chercher de l'aide chez les vivants et les morts afin de survivre, Sam dans le film Ghosts de Jerry Zucker et Jamie dans le film Truly, Madly, Deeply, d'Anthony Minghella ont tout l'air de fantômes recyclés. Ils voyagent dans le temps et dans l'espace mais ne répondent plus à l'image des fantômes de jadis qui cherchaient à sonder les 
profondeurs de la mort et de la solitude. Le fantôme déraciné a trouvé une place au sein de notre culture visuelle, mais, selon Lee Kovacs, le fantôme littéraire attend patiemment dans l'ombre, guettant le moment favorable de sa resurrection.

INDEX

Thèmes : Recensions

\section{AUTEUR}

ANNE LUYAT

Université d'Avignon 